\title{
C. Die Werbeselbstkontrolle als Mittel gegen geschlechtsdiskriminierende Werbung
}

Einen Versuch, gegen geschlechtsdiskriminierende Wirtschaftswerbung vorzugehen, unternimmt der Deutsche Werberat als Selbstkontrollgremium der Werbewirtschaft. Einer Prüfung, ob eine gesetzliche Beschränkung geschlechtsdiskriminierender Wirtschaftswerbung verfassungskonform und mit dem europäischen Primärrecht vereinbar ist, bedarf es nur dann, wenn das angestrebte Ziel nicht auf anderem Wege, insbesondere ohne gesetzliche Regulierung, genauso effektiv erreicht werden kann. Diesen Weg könnte die Werbeselbstkontrolle der Wirtschaft bieten.

\section{Werbeselbstkontrolle in Deutschland}

Zentrales Instrument der Werbeselbstkontrolle in Deutschland ist der Deutsche Werberat. Gedrängt durch eine Resolution des Europarates und um drohenden gesetzlichen Werbebeschränkungen vorzubeugen ${ }^{191}$ wurde er 1972 vom Zentralverband der Deutschen Werbewirtschaft (ZAW) e.V. gegründet und arbeitet seitdem als Organ des ZAW als Konfliktregler zwischen Beschwerdeführenden aus der Bevölkerung und werbenden Firmen und steht als ständiges Ansprechorgan für verbraucherbezogene Probleme mit Werbung im Vorfeld der gesetzlichen Bestimmungen zur Verfügung. ${ }^{192}$ Der ZAW setzt sich als Dachorganisation von vierzig Verbänden aus vier Gruppen zusammen: Werbungtreibende, Werbungdurchführende, Werbeagenturen und Werbeberufe. ${ }^{193}$ Das Präsidium wählt aus seiner Mitte zehn Persönlichkeiten des Wirtschaftszweigs als Entscheidungsgremium des Werberates und kann zusätzliche Fachleute von außen berufen; gegenwärtig sind es drei. Diese insgesamt 13 Fachexpert_innen der werbenden Unternehmen,

191 von Hippel ZRP 1973, 177, 178.

192 Ziff. 1 der Arbeitsgrundsätze des Deutschen Werberates, Deutscher Werberat Jahrbuch 2012, S. 75; ausführlich http://www.werberat.de/aufgaben-und-ziele (abgerufen am 16.12.2013).

193 Ziff. 4 der Arbeitsgrundsätze des Deutschen Werberates, Deutscher Werberat Jahrbuch 2012, S. 76. 
Medien und Agenturen diskutieren und entscheiden anhand der Verhaltensgrundsätze des Werberates über die jeweils von Beschwerden betroffenen Kampagnen; ${ }^{194} 340$ waren es im Jahr 2013. ${ }^{195}$ Auf diese Weise sollen im Rahmen freiwilliger Selbstverantwortung Missstände im Werbewesen festgestellt und beseitigt und Werbung grundsätzlich im Hinblick auf Inhalt, Aussage und Gestaltung weiterentwickelt werden. Neben der Beurteilung einzelner Werbemaßnahmen obliegt es dem Werberat auch, Grundsatzempfehlungen, Leitlinien und Verhaltensregeln zu erstellen. ${ }^{196}$ Über die Entscheidungspraxis des Gremiums erscheinen jährlich die Jahrbücher Deutscher Werberat. Seit 1991 vertritt der Werberat Deutschland in der European Advertising Standards Alliance (EASA), deren Hauptaufgabe es ist, ein Verfahren für die Selbstkontrolle grenzüberschreitender Werbung zu ermöglichen. ${ }^{197}$

\section{Umgang mit geschlechtsdiskriminierender Werbung}

Geschlechtsdiskriminierung ist mit großem Abstand das häufigste Beschwerdemotiv vor dem Deutschen Werberat: Seit 2007 liegt der Anteil beständig zwischen 33 und $42 \% .{ }^{198}$ Während der Vorwurf männerdiskriminierender Werbung konstant im geringen einstelligen Prozentbereich zu finden ist, ${ }^{199}$ verzeichnet der Werberat insgesamt einen Anstieg der Beschwerden über frauendiskriminierende Werbekampagnen - von 2009 auf 2010 um mehr als ein Fünftel ${ }^{200}$ und von 2012 auf 2013 um neun Prozent. ${ }^{201}$ Der Werberat begründete dies einerseits mit der insgesamt gestiegenen Zahl von

194 Ziff. 4 der Arbeitsgrundsätze des Deutschen Werberates, Deutscher Werberat Jahrbuch 2012, S. 76; ausführlich http://www.werberat.de/entscheidungsgremium (abgerufen am 16.12.2013).

195 Deutscher Werberat Jahrbuch 2014, S. 19.

196 Arbeitsgrundsätze des Deutschen Werberates, Deutscher Werberat Jahrbuch 2012, S. $75 \mathrm{f}$.

197 http://www.werberat.de/european-advertising-standards-alliance-easa (abgerufen am 16.12.2013).

198 Deutscher Werberat Jahrbuch 2012, S. 27; Deutscher Werberat Jahrbuch 2011, S. 23.

199 Deutscher Werberat Jahrbuch 2009, S. 27; Deutscher Werberat Jahrbuch 2011, S. 23: In den Jahren 2009 und 2010 kam diese Art der Vorwürfe mit $2 \%$ auf den 12. von 15 Plätzen; Deutscher Werberat Jahrbuch 2012, S. 27: 5\%.

200 Deutscher Werberat Jahrbuch 2011, S. 6 f.

201 Deutscher Werberat Jahrbuch 2014, S. 20. 
Werbekampagnen durch den Ausbau digitaler Netze und mit der mangelnden Professionalität einzelner Firmen. ${ }^{202}$ Andererseits meint er bezüglich der Akzeptanz von Darstellungen in der Werbung einen gesellschaftlichen Wandel „,von weit gefasster Liberalität zu strengeren Formen“ zu erkennen; da grundsätzlich weniger provokant geworben würde, fielen ,erotisch oder gar sexuell aufgeladene Frauenbilder [...] deshalb heute dem Publikum stärker als Kontraste bis hin zu Ärgernissen auf.“203

2011 verneinte der Werberat in $60 \%$ der Beschwerdefälle mit dem Vorwurf der „Frauendiskriminierung“ eine Verletzung der Verhaltensregeln. Die ,relativ hohe Anzahl unbegründeter Beschwerden“ erklärt der Werberat in folgender Weise: ,Individuelle biografische Erlebnisse, kritische Einstellungen zur Liberalität in Sachen Erotik und Sexualität oder Überbesorgnis über den aktuellen Zustand der Gesellschaft suchen Ventile und finden sie unter anderem in der Werbung der Unternehmen. “204

\section{Kriterienkatalog}

Der Deutsche Werberat entscheidet auf Grundlage seiner Verhaltensgrundsätze, welche neben der Veröffentlichung in den Jahrbüchern ${ }^{205}$ auch auf der Homepage 206 einsehbar sind. Die Grundsätze zur Herabwürdigung und Diskriminierung von Personen in der Fassung von 2004 lauten wie folgt: „In der kommerziellen Werbung dürfen Bilder und Texte nicht die Menschenwürde und das allgemeine Anstandsgefühl verletzen. Insbesondere darf Werbung - gerade gegenüber Kindern und Jugendlichen - nicht den Eindruck erwecken, dass bestimmte Personen minderwertig seien oder in Gesellschaft, Beruf und Familie willkürlich behandelt werden können. Vor allem dürfen keine Aussagen oder Darstellungen verwendet werden,

- die Personen wegen ihres Geschlechts, ihrer Abstammung, ihrer Rasse, ihrer Sprache, ihrer Herkunft, ihres Glaubens, ihrer politischen Anschauung, ihres Alters oder ihres Aussehens diskriminieren

202 Deutscher Werberat Jahrbuch 2011, S. 7.

203 Deutscher Werberat Jahrbuch 2011, S. 7.

204 Deutscher Werberat Jahrbuch 2012, S. 26.

205 Deutscher Werberat Jahrbuch 2012, S. 86 f.; Deutscher Werberat Jahrbuch 2014, S. $69 \mathrm{ff}$.

206 http://www.werberat.de/diskriminierung (abgerufen am 16.12.2013). 
- die Gewalt oder die Verharmlosung von Gewalt gegenüber Personen enthalten

- die den Eindruck erwecken, Personen seien käuflich zu erwerben

- die den herrschenden allgemeinen Grundüberzeugungen widersprechen (zum Beispiel durch übertriebene Nacktheit)

- die Personen auf ihre rein sexuelle Funktion reduzieren und/oder deren ständige sexuelle Verfügbarkeit nahelegen

- die pornografischen Charakter besitzen.

Ob ein Verstoß gegen diese Grundsätze vorliegt, hängt insbesondere von folgenden Kriterien ab:

- Eindruck des verständigen Durchschnittsverbrauchers

- Charakter des Mediums

- Art des beworbenen Produkts/der beworbenen Dienstleistung

- aktuell herrschende Auffassung über Sitte, Anstand und Moral in der Gesellschaft

- dargestellte gesellschaftliche Wirklichkeit wie beispielsweise in redaktionellen Teilen der Medien, Film oder Theater.“207

\section{Beschwerdeverfahren}

Das Beschwerdeverfahren kann per Telefon, Email, Post, Fax oder mit Hilfe des Beschwerdeformulars mit Beschwerden über Werbemaßnahmen von Unternehmen an den Deutschen Werberat in Gang gesetzt werden. ${ }^{208}$ Es genügt dafür bereits eine einzige Beschwerde, eine Mindestanzahl von Beschwerden ist nicht nötig. Wenn die Geschäftsführung des Werberates sie nicht als offensichtlich unbegründet zurückweist, leitet sie die Beschwerde nach ihrem Eingang weiter an den Werberat. In diesem Fall wird zunächst die Stellungnahme des betroffenen Unternehmens eingeholt. ${ }^{209}$ Danach entscheidet das Gremium per Mehrheitsbeschluss. Wenn es die Beschwerde nicht verwirft, sondern sich ihr anschließt, fordert der Werberat das Unternehmen zur Korrektur oder der Einstellung der Kampagne auf. Wenn das

207 Grundsätze des Deutschen Werberates zur Herabwürdigung und Diskriminierung von Personen, Deutscher Werberat Jahrbuch 2014, S. 69.

208 Artikel 1, 3 der Verfahrensordnung des Deutschen Werberates, Deutscher Werberat Jahrbuch 2012, S. 78 f.

209 Art. 3, 6, 11 der Verfahrensordnung des Deutschen Werberates, Deutscher Werberat Jahrbuch 2012, S. 79 ff. 
Unternehmen dieser Aufforderung nicht nachkommt, kann der Werberat die Werbung öffentlich rügen (sog. Instrument der Öffentlichen Rüge). ${ }^{210} \mathrm{Da}-$ rüber hinausgehende Mittel, vor allem die zwangsweise Durchsetzung seiner Entscheidung, stehen dem Werberat nicht zur Verfügung. Abschließend teilt die Geschäftsführung des Werberates den Beschwerdeführer_innen schriftlich $^{211}$ und mit Begründung das Ergebnis des Verfahrens mit.

\section{Kritische Betrachtung der Werbeselbstkontrolle}

\section{Vorteile der Werbeselbstkontrolle}

Das System der wirtschaftlichen Selbstkontrolle, hier durch den Werberat, hat Vorteile: ${ }^{212}$ So kann der Werberat unbürokratisch und kostenfrei tätig werden. Dies fördert die Möglichkeit der Bevölkerung, sich ohne großen Aufwand und vor allem auch ohne finanzielle Last jederzeit gegen diskriminierende Werbung zu wehren. Außerdem können die Entscheidungen sehr schnell getroffen werden: ${ }^{213}$ Von der Beschwerde bis zur Beanstandung durch den Werberat vergehen in der Regel nur wenige Tage bis Wochen, was auf gerichtlichem Wege sonst nur im Eilrechtsschutz möglich ist. Schließlich ist der Werberat in der Anwendung, Ausgestaltung und Erweiterung seiner Verhaltensgrundsätze sehr flexibel; sie können jederzeit überarbeitet und durch neue Fallgruppen ergänzt und ausdifferenziert werden. ${ }^{214}$ Auch kann der Werberat das Handeln der Werbetreibenden sehr viel weiter einschränken, als ein Gesetz oder ein Gericht dies könnten. Durch eine konsequente, gut begründete Arbeit kann der Werberat auf das Verständnis der Werbetreibenden und eine Entwicklung zu einer geschlechtergerechteren Werbelandschaft hinwirken.

Fraglich ist, ob er diese Möglichkeiten auch nutzt und tatsächlich geschlechtsdiskriminierender Werbung effektiv entgegenwirkt.

210 Art. 11 Abs. 3; 12 Abs. 2 der Verfahrensordnung des Deutschen Werberates, Deutscher Werberat Jahrbuch 2012, S. $81 \mathrm{f}$.

211 Art. 11 Abs. 3 der Verfahrensordnung des Deutschen Werberates, Deutscher Werberat Jahrbuch 2012, S. 81.

$212 \mathrm{http} / /$ www.werberat.de/verfahren (abgerufen am 16.12.2013).

213 Baum/Langenbucher/Pöttker/Schicha Handbuch zur Medienselbstkontrolle, S. 255, 263.

214 Lehmann GRUR Int 2006, 123, 125, 126 f.; Beckmann WRP 1991, 702, 704: Möglichkeit der Anpassung an gesellschaftliche Trends. 


\section{Problematische Gremienzusammensetzung}

Der Deutsche Werberat besteht aus 13 Mitgliedern. Davon sind zehn männlich und lediglich die drei zusätzlich berufenen Mitglieder weiblich. ${ }^{215}$ Dass diese Zusammensetzung gerade im Hinblick auf das Erkennen von Geschlechtsdiskriminierung ein gewisses Gefahrenpotenzial birgt, belegt eine im Auftrag des Ministeriums für die Gleichstellung von Frau und Mann des Landes Nordrhein-Westfalen angefertigte Studie zur Rezeption von Sexismus und Gewalt im Fernsehen. Grundlage der Analyse war eine repräsentative Telefonbefragung von 1000 Personen im Alter zwischen 14 und 60 Jahren sowie eine Gruppendiskussion mit 127 Männern und Frauen im Alter zwischen 20 und 50 Jahren. Während viele Männer sexistische Fernsehbilder mit dem Argument, sie seien dem wirklichen Leben nachempfunden, rechtfertigten und häufig ähnliche, traditionelle Vorstellungen über Geschlechtsrollen artikulierten, kamen Frauen mehrheitlich zu anderen Ergebnissen, wenn sie die Fernsehbilder mit der Realität verglichen: Sie empfanden eine deutliche Diskrepanz zwischen erlebter Realität und den Fernsehbildern und waren der Ansicht, das Fernsehen hinke der Wirklichkeit, in der Frauen ihr Leben selbständig und vielseitig meistern, um Jahrzehnte hinterher. ${ }^{216}$ Angesichts der Tatsache, dass die Entscheidungen des Werberates per Mehrheitsbeschluss gefällt werden und die männlich sozialisierten Mitglieder stets deutlich in der Überzahl sind, ist eine bestmögliche Effektivität im Kampf gegen geschlechtsdiskriminierende Werbung zumindest fraglich. Wünschenswert wäre ein paritätisch geschlechtergemischtes Gremium. ${ }^{217}$

Neben der ungleichen Geschlechterverteilung unter den Mitgliedern des Werberates ist ein weiterer Punkt zu bedenken: Der Deutsche Werberat besteht ausschließlich aus Vertreter_innen der werbenden Wirtschaft, der Medien, der Kommunikationsagenturen und der Werbeberufe; auch die zusätzlich Berufenen entstammen ausnahmslos der Wirtschaft. Nicht beteiligt werden Vertreter_innen der Öffentlichkeit, wie z.B. stimmberechtigte Laien

$215 \mathrm{http} / /$ www.werberat.de/entscheidungsgremium (abgerufen am 16.12.2013).

216 Röser/Kroll Was Frauen und Männer vor dem Bildschirm erleben, S. II; dass Frauen Werbung häufiger als geschlechtsdiskriminierend beurteilen als Männer, zeigen auch Rossi/Rossi Sex Roles 1985, 1033, 1038.

217 Ähnlich Baum/Langenbucher/Pöttker/Schicha Handbuch zur Medienselbstkontrolle, S. 255, 263, für den ,ein höherer Anteil von Frauen bei den Mitgliedern des Werberates wünschenswert" wäre. 
und/oder Expert_innen. ${ }^{218}$ Diese könnten die Homogenität des Gremiums auflockern und den Blick der übrigen Mitglieder erweitern. Bisher wehrt sich der Werberat gegen eine solche Änderung seiner Zusammensetzung mit dem Hinweis darauf, dass der Werberat eine selbstdisziplinäre Einrichtung sei; dies schließe die Einbeziehung von Vertreter_innen der Öffentlichkeit bereits begrifflich aus. Außerdem sei eine solche Änderung mit zahlreichen Nachteilen, vor allem bezüglich der Schnelligkeit des Entscheidungsprozesses, verbunden. ${ }^{219}$

Dem ist entgegenzuhalten, dass der Begriff, ,Selbstkontrolle“ eine Fremdbeteiligung nicht zwingend ausschließ $\mathrm{t}^{220}$ und auch andere europäische Werberäte eine solchen Zusammensetzung für möglich und sinnvoll halten: So gehören zum Gremium des italienischen Werberates höchstens 15, mindestens aber neun Fachleute aus den Bereichen Recht, Verbraucherprobleme und Kommunikation. ${ }^{221}$ Ähnliche Ansätze finden sich im britischen und dänischen Werberat. 222 Wenn sich ein Selbstkontrollgremium allein aus Personen zusammensetzt, deren Interessen tendenziell homogen sind, steht die Gefahr der Parteilichkeit im Raum. ${ }^{223}$ Einer solchen könnte begegnet werden, indem auch unparteiischen Dritten oder Vertreter_innen der Marktgegenseite eine ins Gewicht fallende Beteiligung an dem entscheidenden Gremium eingeräumt wird. ${ }^{224}$ Dass der Werberat eine freiwillige Selbstkontrolleinrichtung ist, muss dem nicht entgegenstehen: So wie es ihm gestattet ist, die Regeln und Maßstäbe in eigener Regie festzulegen, kann er auch ,die Richter_innen“ bestimmen, welche die Einhaltung jener Regelungen beurteilen sollen. ${ }^{225}$ Die jetzige Zusammensetzung lässt befürchten, dass die

218 Dies ebenfalls kritisierend Gottzmann Möglichkeiten und Grenzen der freiwilligen Selbstkontrolle, S. 202 f.; Baum/Langenbucher/Pöttker/Schicha Handbuch zur Medienselbstkontrolle, S. 255, 264; von Hippel ZRP 1973, 177, 179.

219 Deutscher Werberat Jahrbuch 1991, S. 12.

220 Gottzmann Möglichkeiten und Grenzen der freiwilligen Selbstkontrolle, S. 12 ff., 203.

221 Lehmann GRUR Int 2006, 123, 124.

222 Schricker GRUR 1974, 579, $588 \mathrm{f}$.

223 Schricker GRUR 1974, 579, $588 \mathrm{f}$.

224 Schricker GRUR 1974, 579, 588 f.; Gottzmann Möglichkeiten und Grenzen der freiwilligen Selbstkontrolle, S. $202 \mathrm{f}$.

225 Lehmann GRUR Int 2006, 123, 124 f.; Gottzmann Möglichkeiten und Grenzen der freiwilligen Selbstkontrolle, S. $12 \mathrm{ff}$. 
Objektivität der Entscheidungen nicht garantiert werden kann und die Interessen der Verbraucher_innen außen vor bleiben. ${ }^{226}$

Die Zusammensetzung des Deutschen Werberates lässt folglich in mehrfacher Hinsicht an der Effektivität des Vorgehens gegen geschlechtsdiskriminierende Werbung zweifeln.

3. Unzureichende Anwendung und problematische Ausformulierung der Verhaltensregeln

Ein weiterer zu untersuchender Punkt sind die Verhaltensregeln des Deutschen Werberates.

\section{a) Unzureichende Anwendung der Verhaltensregeln}

Dort heißt es unter anderem, dass keine Aussagen oder Darstellungen verwendet werden dürfen, die Personen wegen ihres Geschlechts diskriminieren, die den Eindruck erwecken, Personen seien käuflich zu erwerben oder die Personen auf ihre rein sexuelle Funktion reduzieren und/oder deren ständige sexuelle Verfügbarkeit nahelegen. ${ }^{227}$ Diese Ansätze werden nicht weiter erläutert; vielmehr soll bei der Auslegung der jeweiligen Werbeaussage unter anderem auf den Eindruck des ,,verständigen Durchschnittsverbrauchers“ und die ,aktuell herrschende Auffassung über Sitte, Anstand und Moral in der Gesellschaft" abgestellt werden. ${ }^{228}$ Nicht erkennbar ist, ob die „aktuell herrschende Auffassung von Sitte, Anstand und Moral“" anhand übergeordneter Kriterien oder anhand der weltanschaulichen Auffassung und des Alltagsverstands der Gremiumsmitglieder bestimmt wird. ${ }^{229}$ Bedenken ergeben sich hier einerseits daraus, dass Entscheidungen über die „gesellschaftliche Moral“ in die Hände eines relativ homogenen 13-köpfigen Gremiums gelegt sind, ${ }^{230}$ die Gesellschaft selbst aber, auch in ihren

226 Lehmann GRUR Int 2006, 123, 125.

227 Grundsätze des Deutschen Werberates zur Herabwürdigung und Diskriminierung von Personen, Deutscher Werberat Jahrbuch 2012, S. 86 f.

228 Grundsätze des Deutschen Werberates zur Herabwürdigung und Diskriminierung von Personen, Deutscher Werberat Jahrbuch 2012, S. 86 f.

229 Dies ebenfalls kritisierend Baum/Langenbucher/Pöttker/Schicha Handbuch zur Medienselbstkontrolle, S. 255, 264.

230 Vgl. dazu die Kritik an der Gremiumszusammensetzung. 
Moralvorstellungen, zunehmend divers und pluralistisch ist. Hinzu kommt, dass sowohl die durchschnittlichen Rezipient_innen als auch die Mitglieder des Werberates alltäglich geschlechtsdiskriminierender Werbung ausgesetzt sind, sie daher als normale, objektive Wirklichkeit hinnehmen und vielfach nur noch krasse Abweichungen ins Negative wahrnehmen. ${ }^{231}$ So stellt der Werberat selbst häufig darauf ab, dass Darstellungen nur die Wirklichkeit zeigten oder scherzhaft gemeint seien: ,[...] die eher spielerisch-provokante Art, mit der das Thema Verführung bzw. Verhältnis Frau-Mann in die Werbemaßnahme eingebettet ist, lässt durchaus den augenzwinkernden Tenor der Darstellung erkennen“232, heißt es zu einer Plakatwerbekampagne für ein Männerdeodorant, welche den auf einem Flugzeugträger stehenden Unterleib einer Frau zeigt. Der Blick der Rezipient_innen wird auf die in Pumps steckenden nackten Beine der Frau hinauf zu ihrem durch knappe Hotpants bekleideten Gesäß gelenkt. Zwischen ihren gespreizten Beinen steht eine überdimensionierte Deodorantdose, worüber ein kleines, scheinbar auf ihre Genitalien zuhaltendes Flugzeug im Landeanflug zu sehen ist.

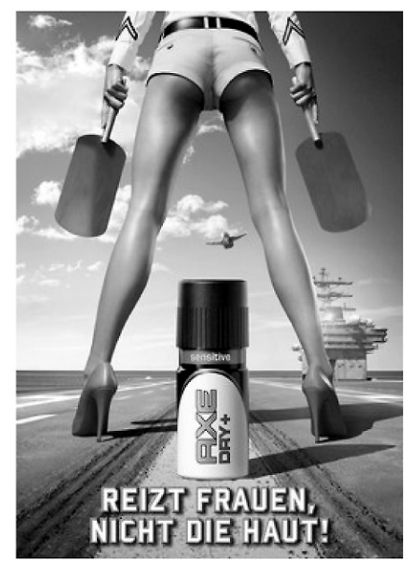

231 Vgl. dazu nur Röser/Kroll Was Frauen und Männer vor dem Bildschirm erleben, S. II; aber auch BGHZ 130, 5, 9 - Busengrapscher: ,[...] die Werbung den Betrachtern gerade nicht mehr als reiner ,Scherz' erscheinen kann, sondern einen Charakter gewinnt, der von dem verbreiteten Einsatz erotischer Anreize oder sexueller Anspielungen in der Werbung deutlich ins Negative abweicht."

232 Veröffentlichung von Teilen einer abschlägigen Antwort des Werberates auf eine Beschwerde: http://maedchenmannschaft.net/tag/axe/ (abgerufen am 16.12.2013). 
Im passend dazu entwickelten Spiel können Spielende ihr „Mannsein unter Beweis stellen“. ${ }^{233}$ In der Spielbeschreibung heißt es: „Zu ,bespielen“ [sic] gilt es einen weiblichen, relativ attraktiven Sergeant, mit dem gezielten Einsatz deines Cursors, in Form einer etwas frivolen Hand. In drei Spielen kannst du zunächst mit einem beherzten Griff an ihren Gürtel einmal klar stellen, wer hier die Befehle gibt. Im zweiten Spiel, der Disco, wird es dann schon etwas lasziver und der Flugzeugträger zur Tanzfläche, bis Madame Sergeant im dritten Game durch einen gezielten Beschuss ihres Hinterteils die Hüllen fallen lässt, endlich ihr Gesicht preisgibt und als Showdown zu einem Flug in die Stratosphäre abhebt. Mission Männlichkeit erfüllt." ${ }^{234}$ Dabei besteht der „gezielte Einsatz des Cursors“ im Schlagen des Gesäßes der Frau. In der Begründung des Werberates, warum keine diskriminierende Werbung vorliege, heißt es: „An keiner Stelle des Spiels wird die Frau in einer unterwürfigen oder herabwürdigen Pose vorgeführt. “235

Das Beispiel lässt vermuten, dass die Entscheidungen des Werberates geprägt sind von den persönlichen Vorstellungen seiner Mitglieder von Diskriminierung, Unterwürfigkeit und Herabwürdigung (von Frauen). Das erweckt den Eindruck, dass sich das Gremium, das darüber befinden soll, ob Werbung geschlechtsdiskriminierend ist, allein auf das eigene Urteil verlässt und wenig Versuche unternimmt, die eigenen Vorstellungen zu Geschlecht und Diskriminierung, welche selbst in hohem Maße durch die Gesellschaft und insbesondere auch mediale Bilder geprägt sind, zu hinterfragen. Was fehlt, ist eine für das Erkennen von Geschlechtsdiskriminierung unentbehrliche Auseinandersetzung mit der Konstruktion von Geschlecht und Geschlechtsrollenstereotypen sowie eine Analyse der Rolle von Medien und Werbung in diesem Zusammenhang. Exemplarisch dafür äußert sich Volker Nickel, bis August 2013 Sprecher des ZAW und Vertreter des Werberates, dahingehend, dass Werbung „Spiegelbild [...] gesellschaftlicher Wirklichkeit“ ist. ${ }^{236}$ Die von „Ideologen“, „Moralisten“, , „Populisten“ und den „Frauen-der-Betroffenheit" ${ }^{\prime 237}$ geführte Diskussion über Sexismus sei lange überholt; die „alte Prüderie“ längst vom Zeitgeist überrollt. Bürgerinnen und

233 http://www.artschoolvets.com/news/2010/05/21/axe-dry-sensitive-campaign-onli ne-game/ (abgerufen am 16.12.2013).

234 Ebenda.

235 Veröffentlichung von Teilen einer abschlägigen Antwort des Werberates auf eine Beschwerde: http://maedchenmannschaft.net/tag/axe/ (abgerufen am 16.12.2013).

236 Nickel Nackte Tatsachen, S. 15.

237 Nickel Nackte Tatsachen, S. 9-11. 
Bürger hätten ein normales Verhältnis zur Lust gefunden und auch das Sexualstrafrecht sei seit 1973 erheblich gelockert. ${ }^{238}$ Außerdem gebe es kaum „nackte“ Frauen in der Werbung. ${ }^{239}$

\section{b) Problematische Ausformulierung der Verhaltensregeln}

Auch die Verhaltensregeln selbst weisen auf eine mangelhafte Auseinandersetzung mit dem Problemfeld Geschlechtsdiskriminierung hin: Zum einen liegen sie noch immer nur in der Fassung von 2004 vor. ${ }^{240}$ Gerade im Bereich Geschlecht, Geschlechtskonstruktion, Gender, Gender-Mainstreaming und Diskriminierungsschutz hat sich seitdem sowohl in der Forschung als auch auf der Gesetzgebungsebene viel getan. Ein seit zehn Jahren unbearbeiteter Verhaltenskatalog zu geschlechtsdiskriminierender Werbung lässt jedenfalls Zweifel daran aufkommen, ob der Werberat alle gesellschaftlichen Entwicklungen bezüglich dieses Themas einbezieht und auch möglichst effektiv umzusetzen versucht.

Neben dem Alter der Verhaltensregeln ist die recht knapp gehaltene Ausformulierung zu kritisieren. Sie erschwert die Ergebnisfindung und erleichtert das Einbrechen privater Ansichten und Empfindungen der entscheidenden Mitglieder. Ein mit fachlicher Hilfe stärker ausdifferenzierter, möglicherweise auch mit Beispielen versehener Katalog könnte der gerade durch die Medien schon existierenden ,objektiven Normalität“ in den Köpfen der Entscheidenden entgegenwirken, sie für Probleme erst sensibilisieren und ihnen die Entscheidung damit erleichtern. Gerade was die umfangreiche Ausformulierung und stetige Verbesserung der Verhaltensregeln angeht, zeigen sich Werberäte anderer Länder, z.B. der österreichische, ${ }^{241}$ deutlich engagierter. Die Entscheidungen des Werberates wären im Falle einer stärkeren Ausformulierung auch für Beschwerdeführende und werbende Un-

238 Nickel Nackte Tatsachen, S. 13.

239 Nickel Nackte Tatsachen, S. 16; jedenfalls verkennt Nickel dabei, dass Sexismus ein Synonym für Geschlechtsdiskriminierung ist und nicht zwingend etwas mit Sexualität und Nacktheit zu tun hat. Zur Definition von geschlechtsdiskriminierender Werbung vgl. oben, B. V., S. 62 ff.

240 Grundsätze des Deutschen Werberates zur Herabwürdigung und Diskriminierung von Personen, Deutscher Werberat Jahrbuch 2014, S. 69.

241 Verhaltensregeln des Österreichischen Werberates zu geschlechterdiskriminierender Werbung, http://www.werberat.or.at/show_4274.aspx (abgerufen am 16.12.2013). 
ternehmen nachvollziehbarer und, vor allem für letztere, auch vorhersehbarer. Diesen Vorteil könnte zusätzlich eine bessere Aufarbeitung der entschiedenen Fälle unterstützen. Auch hier zeigt sich der österreichische Werberat mit einer stets aktuellen, übersichtlichen, vollständigen, chronologischen Sammlung sämtlicher positiver und negativer Entscheidungen inklusive Begründung als gutes Vorbild. ${ }^{242}$

\section{Probleme im Verfahren}

Innerhalb des Verfahrens zeigt sich beim Umgang mit (möglicherweise) geschlechtsdiskriminierender Werbung ein weiterer kritischer Punkt: Vor seiner Entscheidung holt der Werberat die Stellungnahme des jeweiligen Unternehmens ein. Da es für das Unternehmen um die Aufrechterhaltung der aktuellen Werbekampagne geht und damit finanzielle Interessen von zum Teil erheblichem Ausmaß betroffen sind, liegt es im Interesse des Unternehmens, dem Werberat mit einer ausführlichen, wohlüberlegten, ausdifferenzierten Argumentation zugunsten der Unbedenklichkeit der jeweils in der Kritik stehenden Werbekampagne zu antworten. Je nach Überzeugungskraft ist die Stellungnahme des Unternehmens also geeignet, den Verfahrensausgang zu beeinflussen. ${ }^{243}$

Es könnte bereits die Frage gestellt werden, warum den Unternehmen überhaupt die Möglichkeit zur Stellungnahme gewährt wird. Schließlich kommt es im Rahmen der Prüfung, ob eine diskriminierende Werbung vorliegt, nach den Verhaltensgrundsätzen des Werberates auf den Eindruck des verständigen Durchschnittsverbrauchers, nicht aber auf die Intention des werbenden Unternehmens an.

Jedenfalls aber bräuchte es eines Gegengewichtes zu der Stellungnahme des Unternehmens. Der Werberat selbst kann diese Rolle nicht ausfüllen, da er einerseits neutrales Entscheidungsgremium sein soll, andererseits seine Mitglieder sogar selbst aus der Werbebranche stammen. Das Gegengewicht kann daher allein in den Beschwerden der jeweiligen Beschwerdeführenden liegen. Allerdings sind diese nicht zwingend mit argumentativem Talent und fachlichem Wissen zum Thema Geschlechtsdiskriminierung ausgestattet.

242 Aktuelle Beschwerdeliste des Österreichischen Werberates, http://www.werberat. or.at/beschwerdeliste.aspx?tid=3 (abgerufen am 16.12.2013).

243 Darauf hindeutend auch die Untersuchung von Beier Schwachstellen der Werbeselbstkontrolle, S. $42 \mathrm{ff}$. 
Auch ist ihnen nicht unbedingt bewusst, dass ihre Beschwerde zusammen mit der Stellungnahme des Unternehmens neben den Verhaltensgrundsätzen die Entscheidungsgrundlage für den Werberat bildet. Vermutlich ergeben sich private Beschwerden an den Werberat häufig aus einer - manchmal nur schwer begründbaren - Unzufriedenheit mit Werbebildern, auf welche der Werberat aufmerksam gemacht werden und mit denen er sich beschäftigen soll. Die Motivation eines Unternehmens für eine umfangreiche, überzeugende Begründung ist jedoch ungleich größer, geht es hier doch darum, ob das Unternehmen eventuell eine bereits fertiggestellte und bezahlte Werbekampagne ändern oder gar aus dem Verkehr nehmen muss. Ein Gegengewicht ist also zwingend nötig, wenn nicht der Verdacht eines Ungleichgewichts zugunsten der Unternehmen im Raum stehen soll.

Beispiel für eine dieses Problem vermeidende Lösung ist das Verfahren des südafrikanischen Werberates, ${ }^{244}$ der neben der Stellungnahme der Unternehmen stets auch Meinungen Dritter einholt, z.B. von der staatlichen Kommission für Geschlechtergleichheit. Auf diese Stellungnahmen kann das Unternehmen wiederum antworten. Anders als der deutsche Werberat veröffentlicht sein südafrikanisches Pendant neben der eigenen Begründung und Entscheidung auch sämtliche Stellungnahmen im jeweiligen Fall. ${ }^{245}$ Dadurch wird ein auch für die Öffentlichkeit sichtbarer Diskussions- und Entscheidungsprozess möglich. Dem Werberat kann so kaum noch der Vorwurf gemacht werden, er entscheide nur darüber, ob er sich der Stellungnahme des Unternehmens anschließt oder nicht. Vielmehr wird sichtbar, dass der Werberat eine eigene, auf sämtliche geäußerte Stellungnahmen aufbauende Entscheidung trifft.

5. Unzulänglichkeiten des Sanktionssystems und im Rahmen der Durchsetzungsfähigkeit

Im Rahmen der Frage nach der Effektivität des Handelns des Werberates gegen geschlechtsdiskriminierende Werbung sind schließlich das Sanktionssystem und die Durchsetzungsfähigkeit des Gremiums in Augenschein zu nehmen.

244 ASA Procedural Guide Nr. 11, http://www.asasa.org.za/Default.aspx?mnu_id=43 (abgerufen am 16.12.2013).

245 http://www.asasa.org.za/RecentRulings.aspx (abgerufen am 16.12.2013). 
Leistet das betroffene Unternehmen der Beanstandung des Werberates nicht Folge, so kann dieser die Öffentlichkeit in einer öffentlichen Rüge davon unterrichten. ${ }^{246}$ Weitergehende Mittel stehen dem Werberat nicht zu. Als Gremium der wirtschaftlichen Selbstkontrolle haben seine Entscheidungen vor allem empfehlenden Charakter, können also nicht gerichtlich durchgesetzt werden. Auch Bußgelder oder andere Zwangsmaßnahmen werden durch die wirtschaftliche Selbstkontrolle nicht verhängt. Nach Angaben des Werberates genügt in den meisten Fällen die Androhung der Rüge, um die Beanstandung durchzusetzen; in lediglich 4 bis $10 \%$ aller Fälle erfolge die öffentliche Rüge. ${ }^{247}$ Darüber hinaus geht der Werberat davon aus, dass Unternehmen jegliche negative Publicity möglichst verhindern wollen und die meisten sich daher kooperativ zeigen. ${ }^{248}$ Auch Teile der Literatur sind der Ansicht, dass die öffentliche Rüge nur auf den ersten Blick kein wirksames Sanktionsmittel sei, da letztlich alle Unternehmen am Verkauf der Produkte und Dienstleistungen interessiert wären und daher die öffentliche Meinung nicht ignorieren könnten. ${ }^{249}$ Dem ist entgegenzuhalten, dass es immer wieder Unternehmen gibt, die ihre Werbekampagne auch nach einer Rüge nicht einstellen ${ }^{250}$ - sei es, weil finanzielle Gründe im Wege stehen, weil ihnen die öffentliche Wirkung gleichgültig ist oder weil auch negative Öffentlichkeit als nicht unwillkommene Werbung empfunden wird. Dafür spricht, dass jedenfalls bei noch unbekannten Produkten eine absatzsteigernde Wirkung auch durch negative mediale Aufmerksamkeit angenommen wird. ${ }^{251}$ Die Arbeit des Werberates erreicht also dort ihre Grenzen, wo Werbetreibende nicht mit der Selbstkontrolle kooperieren wollen. ${ }^{252}$

$\mathrm{Zu}$ dem Problem der mangelnden Durchsetzbarkeit tritt ein zweiter Aspekt: Das Sanktionssystem des Werberates besteht allein aus der Beanstan-

246 http://www.werberat.de/sanktionen; http://www.werberat.de/bilanz-2012 (jeweils abgerufen am 16.12.2013).

247 Ebenda.

248 Ebenda.

249 Lehmann GRUR Int 2006, 123, 126; Beckmann WRP 1991, 702, 704.

250 Vgl. z.B. den Fall „Geiler Bodenbelag“, http://www.oberberg-heute.de/?act=vp\& cid=1\&pid=9033 (abgerufen am 16.12.2013), das Unternehmen wurde 2009 gerügt, dessen mit der gerügten Werbung beklebte Fahrzeuge waren aber jedenfalls noch im Jahr 2012 im Stadtgebiet Köln zu sehen.

251 Berger/Sorensen/Rasmussen Marketing Intelligence Review 2011, 48 ff.

252 Daher die Entwicklung eines wirksamen Sanktionssystems fordernd: Schricker GRUR 1974, 579, $588 \mathrm{f}$. 
dung und der nur unter Umständen nötigen Rüge. ${ }^{253}$ Ein abgestufteres Sanktionssystem könnte gerade im Bereich geschlechtsdiskriminierender Werbung sinnvoll sein. So könnten unterhalb der Beanstandung Hinweise zu solchen Werbekampagnen ergehen, die Geschlecht auf eine einseitige Art konstruieren. Dies hätte den Vorteil, dass Unternehmen nicht stets ihre komplette Werbekampagne ändern oder ganz beenden müssten, sondern langsam an ein geschlechtersensibles Verständnis herangeführt werden könnten. Ähnlich abgestufte Sanktionssysteme nutzen unter anderem auch der Deutsche Presserat (mit Hinweisen, Missbilligungen, öffentlicher und nichtöffentlicher Rüge) ${ }^{254}$ und der Österreichische Werberat (ebenfalls in Form von Hinweisen, genannt Sensibilisierung) ${ }^{255}$.

\section{Werbeselbstkontrolle statt staatlicher Zwangsmaßnahmen}

An den genannten kritischen Punkten lässt sich vieles verbessern. Doch stellt sich die Frage, ob eine ideal funktionierende Werbeselbstkontrolle ein gesetzliches Verbot geschlechtsdiskriminierender Werbung ersetzen kann.

Als Hauptargument für die Werbeselbstkontrolle wird zum Teil die „Regulierungsmöglichkeit von noch Zulässigem aber dennoch Unerwünschtem“ vorgebracht, ${ }^{256}$ womit insbesondere Geschmacklosigkeiten und Anstößiges gemeint sein sollen, welche die Grenze zu einer ,gegen die Menschenwürde verstoßenden Diskriminierung“ (noch) nicht (eindeutig) überschreiten. ${ }^{257}$ Diese Bereiche unterlägen letztlich Geschmack und Empfindung der betrachtenden Person und seien daher dem Bereich der richterlichen Beurteilung entzogen; die Werbeselbstkontrolle aber könne mit Argumenten der Sitte, Moral und des guten Geschmacks urteilen, da die Befolgung ihrer Entscheidungen auf Freiwilligkeit beruhe und nicht mit Zwang durchgesetzt werden könne. ${ }^{258}$ Die Möglichkeit einer Aufspaltung geschlechtsdiskriminierender Werbemaßnahmen in ,menschenwürdeverletzend oder geschmacklos“" unterliegt jedoch starken Zweifeln. Die hier be-

253 Kritisch dazu auch von Hippel ZRP 1973, 177, 179.

254 Baum/Langenbucher/Pöttker/Schicha Handbuch zur Medienselbstkontrolle, S. 255, 265.

255 Art. 12 Abs. 1 der Verfahrensgrundsätze, http://www.werberat.at/beschwerdeverf ahren.aspx (abgerufen am 16.12.2013).

256 Lehmann GRUR Int 2006, 123, 126.

257 Ebenda.

258 Lehmann GRUR Int 2006, 123, 126. 
handelten Konstellationen sind vor allem im Hinblick auf Art. 3 Abs. 2 S. 1, 2 und Art. 2 Abs. 1 iVm Art. 1 Abs. 1 GG problematisch. ${ }^{259}$ Dass eine objektive rechtliche Erfassung geschlechtsdiskriminierender Werbung möglich ist, deuten in anderen Ländern vorgenommene, nicht allein auf Selbstkontrolle setzende Regulierungen an. ${ }^{260}$

Juristisch begründbare Entscheidungen sind durchaus möglich. Hinzu kommt, dass selbst wenn sich der Werberat zu einer geschlechtergemischten, heterogenen Gruppe entwickelt, die auch auf diskriminierungsspezifisches Fachwissen zurückgreifen kann, die Entscheidung, ob eine Werbung zu beanstanden ist oder nicht, noch immer in den Händen einer Gruppe läge, deren Mitglieder zu einem großen Teil der Werbewirtschaft angehören und damit stets verdächtig sind, bis zu einem gewissen Grad Richter_innen in eigener Sache zu sein. Ob soziale Konflikte - jedenfalls ausschließlich - auf diese Art gelöst werden sollten, erzeugt Bedenken. ${ }^{261}$ Geschlechtsdiskriminierung in der Werbung gefährdet wesentliche verfassungsrechtliche Grundwerte. Die Überwachung über die Einhaltung dieser Grenzen, die Erfüllung verfassungsrechtlicher Standards und Schutzpflichten ist Aufgabe des Staates. Dieser kann sich dabei zwar der Unterstützung der Selbstkontrolle bedienen, darf diese Aufgabe jedoch nie vollständig aus der Hand und in das Belieben einer wirtschaftlichen Selbstkontrolleinrichtung geben. Die Selbstregulierung kann eine gute, ergänzende Methode im Vorgehen gegen geschlechtsdiskriminierende Werbung sein. Die Schutzpflichten des Staates kann sie aber, schon aufgrund der einer Selbstkontrolle immanenten Gefahr der Parteilichkeit, nicht ersetzen. ${ }^{262}$

259 Oben, B. IV., S. 58 ff.; ausführlich dazu unten, D. I. 1., S. 83 ff. (Menschenwürde), D. I. 2., S. 127 ff. (Gleichberechtigungsgebot), D. I. 3., S. 146 ff. (Allgemeines Persönlichkeitsrecht).

260 Vgl. vor allem § 1 Abs. 2 des norwegischen Marktgesetzes, dazu Kur WRP 1995, 790, 791 ff.; ausführlich zu Regulierungen in anderen Ländern unten, F. V. 2., S. $282 \mathrm{ff}$.

261 Kritisch dazu auch Joerges/Teubner/Günther Rechtsverfassungsrecht, S. 295, 308, mwN.

262 Ebenso Schricker/Henning-Bodewig Elemente einer Harmonisierung des Rechts des unlauteren Wettbewerbs in der Europäischen Union, S. 82. 


\section{Ergebnis}

Die Werbeselbstkontrolle ist hilfreich im Kampf gegen geschlechtsdiskriminierende Werbung, lässt jedoch große Lücken. Sie kann unterhalb von Verbotsschwellen ansetzen, schnell und effektiv tätig werden, die entscheidenden Personen der Werbewirtschaft durch konsequente, gut begründete Entscheidungen schulen und so auch tatsächliches Verständnis erzeugen. Selbst dieses Potenzial wird vom Deutschen Werberat bisher aber nicht ausgeschöpft. Vor allem wird schon eines der Hauptprobleme im Zusammenhang mit geschlechtsdiskriminierender Werbung, die Schwierigkeit des Erkennens und Definierens aufgrund sozialer und insbesondere medialer Konditionierung, nicht gesehen und demzufolge auch im Rahmen der Gremienbesetzung und der Ausgestaltung und Anwendung von Verhaltensgrundsätzen nicht einbezogen. Hinzu kommen die mangelnde Durchsetzungsfähigkeit und allgemeine Verbesserungsmöglichkeiten im Sanktionssystem. Die „Privatisierung des juristischen Regelungsmechanismus vermittels eines zwischengeschalteten moralischen Diskurses“263 versagt dort, wo ein moralischer Diskurs nur unzureichend stattfindet.

Aber auch ein ideal funktionierender Werberat kann, schon aufgrund der Gefahr der potenziellen Parteilichkeit der die Entscheidung tragenden Personen die Erfüllung der staatlichen Schutzpflichten letztlich stets nur unterstützen, nicht aber vollständig ersetzen.

263 So Leschke Einführung in die Medienethik, S. 16. 